\title{
Design of modified booth based multiplier with carry pre-computation
}

\author{
Chaitanya CVS ${ }^{1}$, Sundaresan C ${ }^{2}$, P R Venkateswaran ${ }^{3}$, Keerthana Prasad ${ }^{4}$ \\ 1,2,4 School of Information Sciences, Manipal Academy of Higher Education, India \\ ${ }^{3}$ Bharat Heavy Electricals Limited, India
}

\begin{tabular}{l} 
Article Info \\
\hline Article history: \\
Received Oct 11, 2018 \\
Revised Dec 9, 2018 \\
Accepted Dec 22, 2018 \\
\hline
\end{tabular}

\section{Keywords:}

Binary Multiplication

Carry Pre-Computation

Modified Booth Multiplier

Multiplier Architecture

Vedic Multiplier

\begin{abstract}
Arithmetic unit is the most important component of modern embedded computer systems. Arithmetic unit generally includes floating point and fixedpoint arithmetic operations and trigonometric functions. Multipliers units are the most important hardware structures in a complex arithmetic unit. With increase in chip frequency, the designer must be able to find the best set of trade-offs. The ability for faster computation is essential to achieve high performance in many DSP and Graphic processing algorithms and is why there is at least one dedicated Multiplier unit in all of the modern commercial DSP processors. Tremendous advances in VLSI technology over the past several years resulted in an increased need for high speed multipliers and compelled the designers to go for trade-offs among speed, power consumption and area. A novel modified booth multiplier design for high speed VLSI applications using pre-computation logic has been presented in this paper. The proposed architecture modeled using Verilog HDL, simulated using Cadence NCSIM and synthesized using Cadence RTL Compiler with 65nm TSMC library.The proposed multiplier architecture is compared with the existing multipliers and the results show significant improvement in speed and power dissipation.
\end{abstract}

Copyright (c) 2019 Institute of Advanced Engineering and Science. All rights reserved.

\section{Corresponding Author:}

Chaitanya CVS

School of Information Sciences,

Manipal Academy of Higher Education,

Manipal 576104, Karnataka, India.

Email: chaitanya.cvs@manipal.edu

\section{INTRODUCTION}

Processors are important part of integrated circuits (IC). Large numbers of functionalities are packed in an IC thanks to tremendous growth in density of integration in recent times. As the number of functions increases, the need for computation also grows. With the advent of new process technologies, shrinking of feature size and availability of modern CAD tools, a development of complex integrated circuits for various applications is possible. Examples of such applications include digital signal processing [1], [2], mobile computations and communications, multimedia applications and processing required for scientific computing and applications etc. The speed and efficiency of processor in such IC is very crucial for meeting the requirements of the applications supported by the IC. The speed of processor and efficiency of processor inturn depends upon an arithmetic logic unit [3] which is considered as the main computational unit of the processor.

Moreover, the multiplier units [4] are the most important hardware structures in a complex arithmetic unit. The multiplier units are capable of performing operations on operands of various data types such as calculating running sum of products. As multiplication is a crucial arithmetic operation in processors [5] and digital computer systems, multipliers are the core building block for many algorithms in a wide variety of computing applications. Although multipliers are main arithmetic components used for processing scientific data, the excessive power consumption and delay attracts attention from the research community. Usually, 
multiple arithmetic cores working in parallel are used so as to process large amounts of data with relatively low power and delay.

However, in the high-speed processors which are operating at higher clock frequencies, the existing multiplier takes more delay for execution of the instructions. The existing multiplier units that consume more power are not suitable to be incorporated in the processors which are used in wireless and portable devices. Thus, power savings is an important area for improvement.

In order to address the low power computation along with high performance, a new approach to multiplier design based on ancient Vedic Mathematics has been explored. The mathematical operations using Vedic mathematics are very fast and require less hardware. This aspect of Vedic mathematics can be utilized to increase the computational speed of multipliers. This paper describes the design and implementation of a Vedic multiplier based on Urdhva-Tiryagbhyam Sutra [6]-[9]. The number of steps required to perform a multiplication operation by using UrdhvaTiryagbhyam Sutra are considerably less compared to the conventional multiplication techniques. In this paper, we have further explored a novel method to enhance the speed of a Vedic multiplier by pre-computing the carries which are used during summation of partial products. The implementation of pre-computation logic using multiplexer-based carry-look ahead logic and XOR logic resulted in reduction of delay. The proposed carry pre-computation is used along with Modified Booth methodology resulted in further reduction of delay in performing multiplication operation.

The structure of the paper is divided as follows: The methodology and the architecture of the proposed multipliers are given in section 2. Results are presented in section 3. Finally, conclusion is given in section 4.

\section{RESEARCH METHOD}

\subsection{Carry Pre-Computation Based Binary Multiplier}

An 8-bit Binary Vedic Multiplier has been proposed with A and B as inputs and P as the final 16-bit product. The block diagram for 8-bit multiplication is shown in Figure 1. In the proposed multiplier the operands A and B are divided into Higher and Lower parts with 4-bits each.

$$
\begin{aligned}
& \mathrm{A}=\{\mathrm{AH}, \mathrm{AL}\} \\
& \mathrm{B}=\{\mathrm{BH}, \mathrm{BL}\}
\end{aligned}
$$

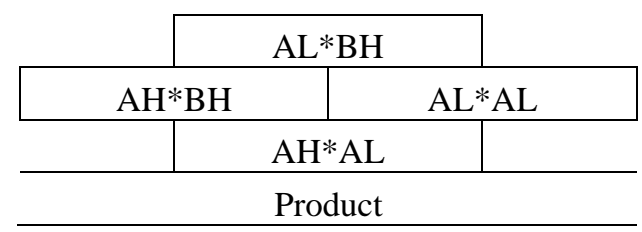

Figure 1. Block Diagram of 8-bit Multiplication

In this type of multiplier an 8-bit Binary multiplication is realized using 4-bit binary vedic multiplication using carry pre-computation logic shown in Figure 2, where $A_{3}, A_{2}, A_{1}, A_{0} \& B_{3}, B_{2}, B_{1}, B_{0}$ are 4-bit binary inputs and $\mathrm{P}_{7}, \mathrm{P}_{6}, \mathrm{P}_{5}, \mathrm{P}_{4}, \mathrm{P}_{3}, \mathrm{P}_{2}, \mathrm{P}_{1}, \mathrm{P}_{0}$ are the binary output bits.

The partial product generator is the first block of the multiplier to which the 4-bit multiplicand and multiplier are given as inputs. At this juncture, the multiplication technique used is Urdhva-Tiryagbhyam. The 4-bit multiplication results in a total of 16 partial products $\left(\mathrm{pp}_{1}-\mathrm{pp}_{16}\right)$. The result of multiplying any one binary bit with another is either a zero or a one which is simply the logic of ANDing of the two bits.

The second stage in the block diagram is the carry generation circuit. Here, we have integrated precomputation logic along with the Urdhva-Tiryagbhyam multiplication technique. The carry equations are generated separately for each column of partial products and the inputs for these equations are taken from the previous column. The equations for pre-computed carries are given.

$$
\begin{aligned}
& \mathrm{c}_{2}=\mathrm{pp}_{5} \& \mathrm{pp}_{2} ; \\
& \mathrm{c}_{3} \mathrm{t}_{1}=\left(\mathrm{pp}_{6} \& \mathrm{pp}_{3}\right) \mid\left(\mathrm{pp}_{9} \&\left(\mathrm{pp}_{3} \mid \mathrm{pp}_{6}\right)\right) ; \\
& \mathrm{c}_{3} \mathrm{t}_{2}=\left(\mathrm{pp}_{9} \& \sim \mathrm{pp}_{6}\right)\left|\left(\mathrm{pp}_{3} \& \sim \mathrm{pp}_{9}\right)\right|\left(\sim \mathrm{pp}_{3} \& \mathrm{pp}_{6}\right) ;
\end{aligned}
$$


$\mathrm{c}_{31}=\mathrm{c}_{2} ? \mathrm{c}_{3} \mathrm{t}_{2}: \mathrm{c}_{3} \mathrm{t}_{1}$

$\mathrm{c}_{32}=\mathrm{pp}_{2} \& \mathrm{pp}_{5} \& \mathrm{pp}_{3} \& \mathrm{pp}_{6} \& \mathrm{pp}_{9}$

$\mathrm{c}_{41} \mathrm{t}_{1}=\mathrm{pp}_{13} ?\left(\left(\mathrm{pp}_{10} \& \sim \mathrm{pp}_{7}\right)\left|\left(\mathrm{pp}_{4} \& \sim \mathrm{pp}_{10}\right)\right|\left(\sim \mathrm{pp}_{4} \& \mathrm{pp}_{7}\right)\right):\left(\left(\mathrm{pp}_{7} \& \mathrm{pp}_{4}\right) \mid\right.$

$\left.\left(\mathrm{pp}_{10} \&\left(\mathrm{pp}_{4} \mid \mathrm{pp}_{7}\right)\right)\right)$;

$\mathrm{c}_{41} \mathrm{t}_{2}=\mathrm{pp}_{13} ?\left(\left(\sim \mathrm{pp}_{7} \& \sim \mathrm{pp}_{4}\right) \mid\left(\sim \mathrm{pp}_{10} \&\left(\sim \mathrm{pp}_{4} \mid \sim \mathrm{pp}_{7}\right)\right)\right):\left(\left(\sim \mathrm{pp}_{7} \& \mathrm{pp}_{4}\right)\left|\left(\mathrm{pp}_{10} \& \sim \mathrm{pp}_{4}\right)\right|\right.$

$\left.\left(\sim \mathrm{pp}_{10} \& \mathrm{pp}_{7}\right)\right)$;

$\mathrm{c}_{41}=\mathrm{c}_{31} ? \mathrm{c}_{41} \mathrm{t}_{2}: \mathrm{c}_{41} \mathrm{t}_{1}$

$\mathrm{c}_{42}=\left(\left(\mathrm{c}_{31} \& \mathrm{pp}_{13}\right) \&\left(\left(\mathrm{pp}_{10} \&\left(\mathrm{pp}_{7} \mid \mathrm{pp}_{4}\right)\right) \mid\left(\mathrm{pp}_{7} \& \mathrm{pp}_{4}\right)\right)\right) \mid\left(\left(\mathrm{pp}_{10} \& \mathrm{pp}_{7} \& \mathrm{pp}_{4}\right) \&\right.$

$\left.\left(\mathrm{c}_{31} \mid \mathrm{pp}_{13}\right)\right)$;

$\mathrm{c}_{51} \mathrm{t}_{1}=\mathrm{c}_{32} ?\left(\left(\mathrm{pp}_{14} \& \sim \mathrm{pp}_{11}\right)\left|\left(\mathrm{pp}_{8} \& \sim \mathrm{pp}_{14}\right)\right|\left(\sim \mathrm{pp}_{8} \& \mathrm{pp}_{11}\right)\right):\left(\left(\mathrm{pp}_{11} \& \mathrm{pp}_{8}\right) \mid\right.$

$\left.\left(\mathrm{pp}_{14} \&\left(\mathrm{pp}_{8} \mid \mathrm{pp}_{11}\right)\right)\right)$;

$\mathrm{c}_{51} \mathrm{t}_{2}=\mathrm{c}_{32} ?\left(\left(\sim \mathrm{pp}_{11} \& \sim \mathrm{pp}_{8}\right) \mid\left(\sim \mathrm{pp}_{14} \&\left(\sim \mathrm{pp}_{8} \mid \sim \mathrm{pp}_{11}\right)\right)\right):\left(\left(\sim \mathrm{pp}_{11} \& \mathrm{pp}_{8}\right)\left|\left(\mathrm{pp}_{14} \& \sim \mathrm{pp}_{8}\right)\right|\right.$

$\left.\left(\sim \mathrm{pp}_{14} \& \mathrm{pp}_{11}\right)\right)$;

$\mathrm{c}_{51}=\mathrm{c}_{41} ? \mathrm{c}_{51} \mathrm{t}_{2}: \mathrm{c}_{51} \mathrm{t}_{1}$

$\mathrm{c}_{52}=\left(\left(\mathrm{c}_{41} \& \mathrm{c}_{32}\right) \&\left(\left(\mathrm{pp}_{14} \&\left(\mathrm{pp}_{11} \mid \mathrm{pp}_{8}\right)\right) \mid\left(\mathrm{pp}_{11} \& \mathrm{pp}_{8}\right)\right)\right) \mid\left(\left(\mathrm{pp}_{14} \& \mathrm{pp}_{11} \& \mathrm{pp}_{8}\right) \&\right.$

$\left.\left(c_{41} \mid c_{32}\right)\right)$;

$\mathrm{c}_{6} \mathrm{t}_{1}=\left(\mathrm{pp}_{12} \& \mathrm{pp}_{15}\right) \mid\left(\mathrm{c}_{42} \&\left(\mathrm{pp}_{12} \mid \mathrm{pp}_{15}\right)\right)$

$\mathrm{c}_{6} \mathrm{t}_{2}=\left(\mathrm{c}_{42} \& \sim \mathrm{pp}_{15}\right)\left|\left(\mathrm{pp}_{12} \& \sim \mathrm{c}_{42}\right)\right|\left(\mathrm{pp}_{15} \& \sim \mathrm{pp}_{12}\right)$

$\mathrm{c}_{61}=\mathrm{c}_{51} ? \mathrm{c}_{6} \mathrm{t}_{2}: \mathrm{c}_{6} \mathrm{t}_{1}$

$\mathrm{c}_{62}=\mathrm{c}_{51} \& \mathrm{c}_{42} \& \mathrm{pp}_{12} \& \mathrm{pp}_{15}$

$c_{71}=\left(c_{52} \& p p_{16}\right) \mid\left(c_{61} \&\left(c_{52} \mid p_{16}\right)\right) ;$

\begin{tabular}{llllllll} 
& & $\mathrm{A}_{3}$ & $\mathrm{~A}_{2}$ & $\mathrm{~A}_{1}$ & $\mathrm{~A}_{0}$ & & \\
& $\mathrm{X}$ & $\mathrm{B}_{3}$ & $\mathrm{~B}_{2}$ & $\mathrm{~B}_{1}$ & $\mathrm{~B}_{0}$ & & \\
\hline & & & & $\mathrm{pp}_{4}$ & $\mathrm{pp}_{3}$ & $\mathrm{pp}_{2}$ & $\mathrm{pp}_{1}$ \\
& & & $\mathrm{pp}_{8}$ & $\mathrm{pp}_{7}$ & $\mathrm{pp}_{6}$ & $\mathrm{pp}$ & \\
& & $\mathrm{pp}_{12}$ & $\mathrm{pp}_{11}$ & $\mathrm{pp}_{10}$ & $\mathrm{pp}_{9}$ & & \\
& $\mathrm{pp}_{16}$ & $\mathrm{pp}_{15}$ & $\mathrm{pp}_{14}$ & $\mathrm{pp}_{13}$ & & & \\
\hline & & & $\mathrm{c}_{32}$ & $\mathrm{c}_{31}$ & $\mathrm{c}_{2}$ & & \\
& & $\mathrm{c}_{42}$ & $\mathrm{c}_{41}$ & & & & \\
& $\mathrm{c}_{52}$ & $\mathrm{c}_{51}$ & & & & & \\
$\mathrm{c}_{62}$ & $\mathrm{c}_{61}$ & & & & & & \\
$\mathrm{c}_{71}$ & & & & & & & \\
\hline $\mathrm{P}_{8}$ & $\mathrm{P}_{7}$ & $\mathrm{P}_{6}$ & $\mathrm{P}_{5}$ & $\mathrm{P}_{4}$ & $\mathrm{P}_{3}$ & $\mathrm{P}_{2}$ & $\mathrm{P}_{1}$
\end{tabular}

Figure 2. Carry Pre-Computation Based Multiplier

The architecture of the 4-bit multiplier can be understood from the block diagram as shown in Figure 3. 


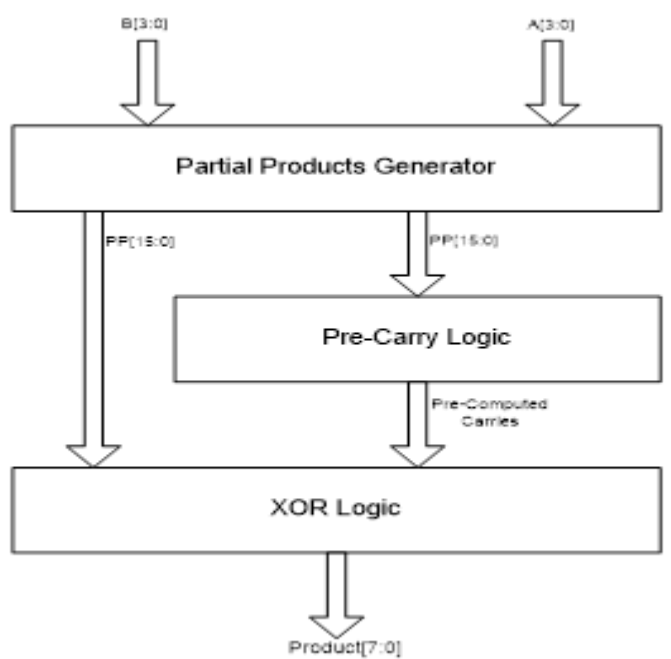

Figure 3. Architecture of Carry Pre-Computation based Multiplier

The third stage in the block diagram involves the use of XOR logic for the partial products and carry generated in each column. The output of this stage gives the final 16-bit product which is obtained in a parallel mechanism instead of sequential mechanism. The products of $\mathrm{AL} * \mathrm{BL}, \mathrm{AH} * \mathrm{BL}, \mathrm{AL} * \mathrm{BH}, \mathrm{AH} * \mathrm{BH}$ are determined using above 4-bit carry pre-computation-based multiplier and the results of all sub multipliers are added to determine the final product. The block of the 8-bit multiplier as shown in Figure 4.

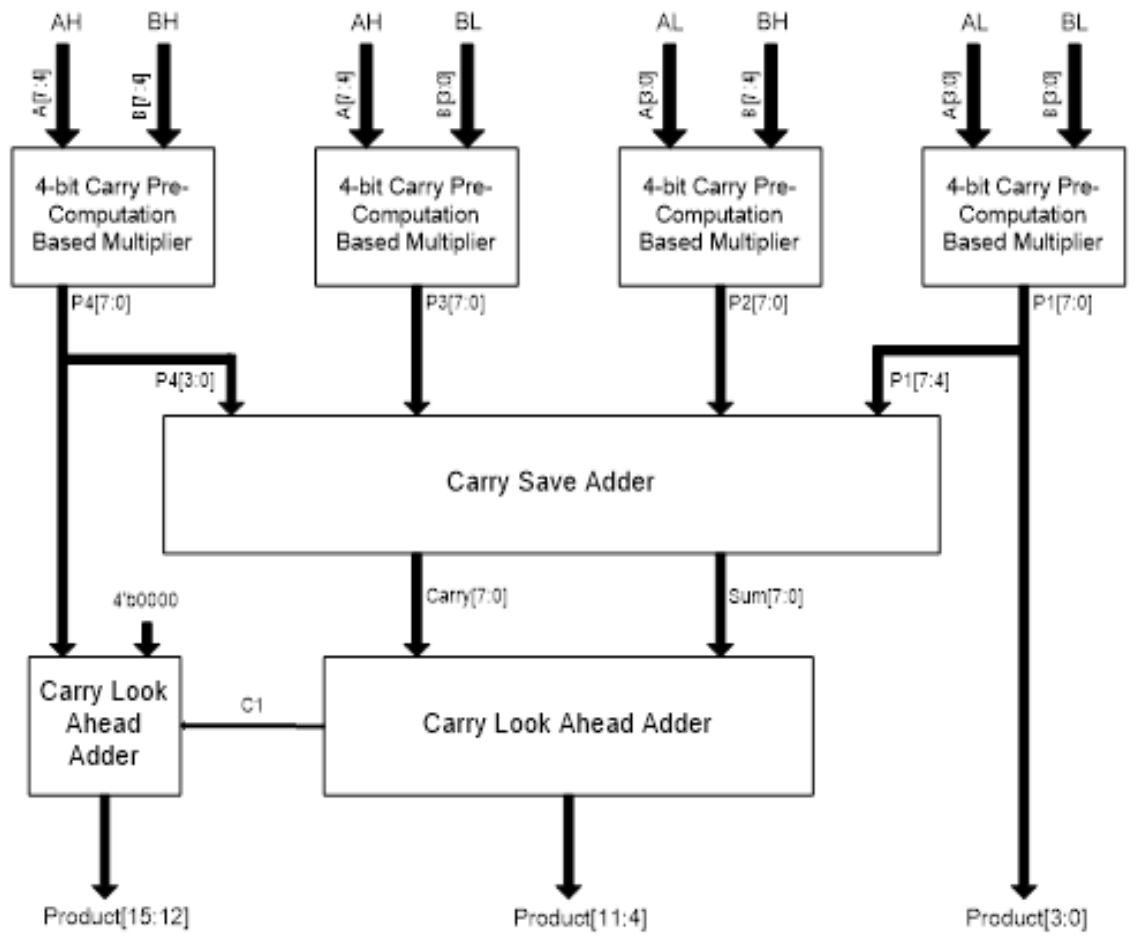

Figure 4. Block Diagram of 8-bit Multiplier Using 4-bit Carry Pre-Computation Based Multiplier

\subsection{Modified Booth Based Binary Multiplier with Carry Pre-Computation}

An 8-bit Binary Modified Booth based multiplier with carry pre-computation has been proposed. The architecture of the 8-bit multiplier can be understood from the block diagram as shown in Figure 5. 


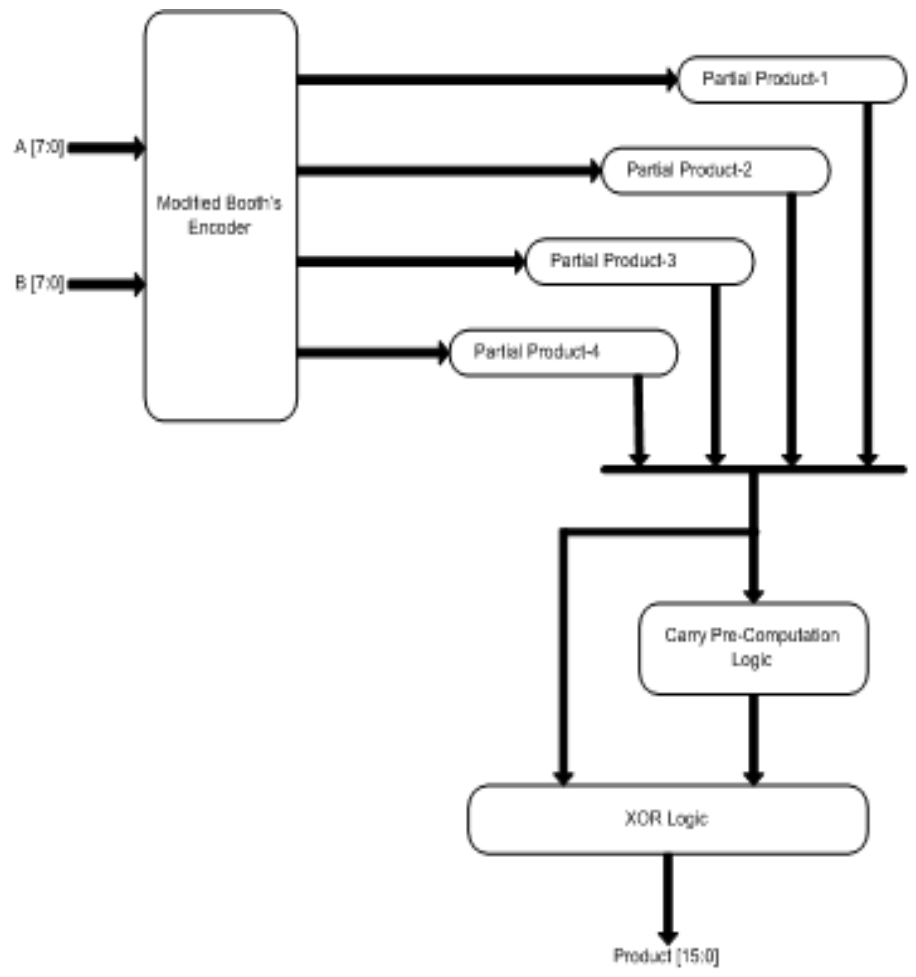

Figure 5. Modified Booth Multiplier with Carry Pre-Computation Logic

The modified booth encoding is the first block of the multiplier to which the 8-bit multiplicand and multiplier are given as inputs. The modified booth encoder generates 4 partial products based on the following Table 1.

Considering that both inputs $\mathrm{A}$ and $\mathrm{B}$ are of 8 bits. As inputs are 8 bits, four partial products will be generated as shown in Figure 6.

Table 1. Modified booth encoding

\begin{tabular}{llll}
\hline $\mathrm{Xi}+1$ & $\mathrm{Xi}$ & $\mathrm{Xi}-1$ & Action \\
\hline 0 & 0 & 0 & $0 \times \mathrm{Y}$ \\
0 & 0 & 1 & $1 \times \mathrm{Y}$ \\
0 & 1 & 0 & $1 \times \mathrm{Y}$ \\
0 & 1 & 1 & $2 \times \mathrm{Y}$ \\
1 & 0 & 0 & $-2 \times \mathrm{Y}$ \\
1 & 0 & 1 & $-1 \times \mathrm{Y}$ \\
1 & 1 & 0 & $-1 \times \mathrm{Y}$ \\
1 & 1 & 1 & $0 \times \mathrm{Y}$ \\
\hline
\end{tabular}

\begin{tabular}{|l|l|l|l|l|l|l|l|l|l|l|l|l|l|l|l|l|}
15 & 14 & 13 & 12 & 11 & 10 & 9 & 8 & 7 & 6 & 5 & 4 & 3 & 2 & 1 & 0 \\
\hline $\mathrm{s} 1$ & $\mathrm{~s} 1$ & $\mathrm{~s} 1$ & $\mathrm{~s} 1$ & $\mathrm{~s} 1$ & $\mathrm{~s} 1$ & $\mathrm{~s} 1$ & $\mathrm{p} 18$ & $\mathrm{p} 17$ & $\mathrm{p} 16$ & $\mathrm{p} 15$ & $\mathrm{p} 14$ & $\mathrm{p} 13$ & $\mathrm{p} 12$ & $\mathrm{p} 11$ & $\mathrm{p} 10$ \\
\hline $\mathrm{s} 2$ & $\mathrm{~s} 2$ & $\mathrm{~s} 2$ & $\mathrm{~s} 2$ & $\mathrm{~s} 2$ & $\mathrm{p} 28$ & $\mathrm{p} 27$ & $\mathrm{p} 26$ & $\mathrm{p} 25$ & $\mathrm{p} 24$ & $\mathrm{p} 23$ & $\mathrm{p} 22$ & $\mathrm{p} 21$ & $\mathrm{p} 20$ & & \\
\hline $\mathrm{s} 3$ & $\mathrm{~s} 3$ & $\mathrm{~s} 3$ & $\mathrm{p} 38$ & $\mathrm{p} 37$ & $\mathrm{p} 36$ & $\mathrm{p} 35$ & $\mathrm{p} 34$ & $\mathrm{p} 33$ & $\mathrm{p} 32$ & $\mathrm{p} 31$ & $\mathrm{p} 30$ & & & & \\
\hline $\mathrm{s} 4$ & $\mathrm{p} 48$ & $\mathrm{p} 47$ & $\mathrm{p} 46$ & $\mathrm{p} 45$ & $\mathrm{p} 44$ & $\mathrm{p} 43$ & $\mathrm{p} 42$ & $\mathrm{p} 41$ & $\mathrm{p} 40$ & & & & & & \\
\hline $\mathrm{c} 132$ & $\mathrm{c} 122$ & $\mathrm{c} 112$ & $\mathrm{c} 102$ & $\mathrm{c} 92$ & $\mathrm{c} 82$ & $\mathrm{c} 72$ & $\mathrm{c} 62$ & $\mathrm{c} 52$ & $\mathrm{c} 42$ & $\mathrm{c} 41$ & $\mathrm{c} 3$ & $\mathrm{c} 2$ & & & \\
$\mathrm{c} 141$ & $\mathrm{c} 131$ & $\mathrm{c} 121$ & $\mathrm{c} 111$ & $\mathrm{c} 101$ & $\mathrm{c} 91$ & $\mathrm{c} 81$ & $\mathrm{c} 71$ & $\mathrm{c} 61$ & $\mathrm{c} 51$ & & & & & & \\
\hline
\end{tabular}

Figure 6. Partial Products of Modified Booth Multiplier 
Partial product $-1:$ p10 to p18 and $\mathrm{s} 1$ is the sign extension Partial product -2 : p20 to p28 and s2 is the sign extension Partial product $-3: \mathrm{p} 30$ to $\mathrm{p} 38$ and $\mathrm{s} 3$ is the sign extension Partial product $-4: \mathrm{p} 40$ to $\mathrm{p} 48$ and $\mathrm{s} 4$ is the sign extension

In Figure,

c2 is carry generated from column 2

c3 is carry generated from column 3

c41 and c42 is carries generated from column 4

c51 and c52 is carries generated from column 5

c61 and c62 is carries generated from column 6

c71 and c72 is carries generated from column 7

c81 and c82 is carries generated from column 8

c91 and c92 is carries generated from column 9

c101 and c102 is carries generated from column 10

c111 and c112 is carries generated from column 11

c121 and c122 is carries generated from column 12

c131 and c132 is carries generated from column 13

c141 is carry generated from column 14

The second stage in the block diagram is the carry generation circuit. Here, we have integrated precomputation logic along with the modified booth multiplication technique. The carry values are generated separately for each column of partial products and the inputs for these equations are taken from the previous column. The carry pre-computation logic discussed in section 2.1 is used to generate the values. The third stage in the block diagram involves the use of XOR logic for the partial products and carry generated in each column. The output of this stage gives the final 16-bit product which is obtained in a parallel mechanism instead of sequential mechanism. In proposed multiplier, as Carry Pre-Computation unit computes all the carries in parallel using carry look ahead logic and remove dependencies between columns, the total time required to generate the product will be reduced.

\section{RESULTS AND ANALYSIS}

The proposed architecture modeled using Verilog HDL, simulated using Cadence NCSIM and synthesized using Cadence RTL Compiler with 65nm TSMC library. Different implementation methodology has been taken and implemented in same technological environment and then compared the performance parameters. For the comparison point of view the ideas have been considered from the references and simulated and performance parameters were computed using the same MOSFET technology file. Input data was taken in a regular fashion for experimental purpose. The delay and the power measured using the worst-case pattern and from the output where the delay is maximum.

It is observed that the proposed carry pre-computation-based multiplier and modified booth multiplier with carry pre-computation based offered substantial reduction of propagation delay and total power consumptions. From Table 1 and Table 2, it can be observed that the proposed carry pre-computation based multiplier design offered $\sim 23 \%, \sim 64 \%, \sim 57 \%, \sim 83 \%$ when compared with array multiplier, wallace multiplier, column based multiplier, and Nikhilam based based multipliers respectively, and modified booth multiplier with carry pre-computation offered $\sim 83 \%, \sim 92 \%, \sim 91 \%, \sim 97 \%$ when compared with array multiplier, wallace, column based, and Nikhilam based multipliers respectively.

Table 2. Summary of synthesis results of 8-bit multiplier architectures

\begin{tabular}{|c|c|c|c|c|c|c|}
\hline S. No & Architecture (8-bit) & $\begin{array}{l}\text { Delay } \\
\text { (ns) }\end{array}$ & $\begin{array}{c}\text { Dynamic } \\
\text { Power }(\mathrm{uW})\end{array}$ & $\begin{array}{l}\text { Static Power } \\
\quad(\mathrm{uW})\end{array}$ & $\begin{array}{l}\text { Total Power } \\
(\mathrm{uW})\end{array}$ & $\begin{array}{l}\text { Power-Delay } \\
\text { Product }(\mathrm{pJ})\end{array}$ \\
\hline 1 & Array Based Multiplier [9] & 1.5 & 15.09 & 6 & 21.09 & 31.63 \\
\hline 2 & Wallace Based Multiplier [2] & 1.2 & 6.27 & 49.913 & 56.184 & 67.42 \\
\hline 4 & Nikhilam Based Multiplier [7] & 3.2 & 42.56 & 4.3 & 46.86 & 149.95 \\
\hline 5 & Pre-Computation Based Multiplier & 0.75 & 25.77 & 7.45 & 33.23 & 24.23 \\
\hline 6 & $\begin{array}{l}\text { Modified Booth's Multiplier with } \\
\text { Carry Pre-Computation }\end{array}$ & 0.45 & 9.4 & 3.99 & 13.39 & 6.02 \\
\hline
\end{tabular}


From the Table 2 and Table 3, it can be observed that Modified Booth based multiplier with carry precomputation consumes less delay when compared to carry pre-computation-based multiplier with the delay tradeoff. Proposed Modified Booth based multiplier with carry pre-computation gave the better power-delay product when compared to proposed carry pre-computation-based multiplier and existing multiplier from literature.

Table 3. Summary of synthesis results of 16-bit multiplier architectures

\begin{tabular}{|c|c|c|c|c|c|c|}
\hline S. No & Architecture (16-bit) & Delay (ns) & $\begin{array}{c}\text { Dynamic Power } \\
(\mathrm{uW})\end{array}$ & $\begin{array}{l}\text { Static Power } \\
(\mathrm{uW})\end{array}$ & $\begin{array}{c}\text { Total Power } \\
\text { (uW) }\end{array}$ & $\begin{array}{l}\text { Power- } \\
\text { Delay } \\
\text { Product }\end{array}$ \\
\hline 1 & Array Based Multiplier [9] & 2.89 & 30.18 & 12 & 42.18 & 121.90 \\
\hline 3 & Column Based Multiplier [6] & 3.82 & 52.48 & 5.4 & 57.88 & 221.10 \\
\hline 4 & Nikhilam Based Multiplier [7] & 5.96 & 80.65 & 8.1 & 88.75 & 528.95 \\
\hline 5 & Pre-Computation Based Multiplier & 1.4 & 51.54 & 14.9 & 66.44 & 93.01 \\
\hline
\end{tabular}

\section{CONCLUSION}

In this paper, a Vedic mathematics-based multiplier has been proposed which uses Carry precomputation and operand decomposition methodology. The proposed architectures combine the benefits of Vedic method and parallel pre-computation of carries thereby resulting in reduction of power-delay product. The propagation delay of carry pre-computation-based multiplier for calculation of 8 bit and 16-bit multiplication was $0.75 \mathrm{~ns}$ and $1.4 \mathrm{~ns}$ while power consumption was $33.23 \mathrm{uW}$ and $66.44 \mathrm{uW}$. The propagation delay of modified booth multiplier with carry pre-computation for calculation of 8 bit and 16-bit multiplication was $0.45 \mathrm{~ns}$ and $0.84 \mathrm{~ns}$ while power consumption was $13.39 \mathrm{uW}$ and $25.53 \mathrm{uW}$. The delay of multiplication was decreased by $\sim 85 \%$ and power consumption were reduced by $\sim 88 \%$ when compared to Nikhilam based Vedic multiplier.

\section{REFERENCES}

[1] Xiangui Kang, AnjiePeng, XianyuXu, Xiaochun Cao, "Performing Scalable Lossy Compression on Pixel Encrypted Images," EURASIP Journal on Image and Video Processing, pp. 1-6, 2013.

[2] Nikolay Ponomarenko, Sergey Krivenko, Vladimir Lukin, Karen Egiazarian, Jaakko T., Astola, "Lossy Compression of Noisy Images Based on Visual Quality: A Comprehensive Study, " EURASIP Journal on Advances in Signal Processing, pp. 1-13, 2010.

[3] L.-K. Wang, M. A. Erle, C. Tsen, E. M. Schwarz, and M. J. Schulte, "A survey of hardware designs for decimal arithmetic," IBM Journal of Research and Development, vol. 54(2), pp. 8:1-8:15, 2010.

[4] M. Jeevitha, R. Muthaiah, P. Swaminathan, "Efficient Multiplier Architecture in VLSI Design," Journal of Theoretical and Applied Information Technology, vol. 38(2), pp. 196-201.2, 2012.

[5] J. R. Boddie, G. T. Daryanani, I. I. Eldumiati, R. N, Gadenz, J. S. Thompson, S. M. Walters, "Digital Signal Processor: Architecture and Performance," Bell System Technical Journal, vol. 60(7), pp. 1449-1462, 1981.

[6] BharatiKrsnaTirthaji, V. S Agrawala, "Vedic Mathematics", 13th Edition, Motilal Banarsidass, 2010.

[7] P. Saha, A. Banerjee, A. Dandapat, and P. Bhattacharyya, "ASIC design of a high-speed low power circuit for factorial calculation using ancient Vedic mathematics, " ELSEVIER Microelectronics Journal, vol. 42(12), pp. 13431352, Dec 2011.

[8] MD. Belal Rashid, Balaji B.S and Prof. M.B. Anandaraju, "VLSI Design and Implementation of Binary Multiplier based on UrdhvaTiryagbhyam Sutra with reduced Delay and Area," International Journal of Engineering Research and Technology, vol. 6(2), pp. 269-278, Mar 2013.

[9] Ko-Chi Kuo, Chi-Wen Chou, "Low Power and High-Speed Multiplier Design with Row Bypassing and Parallel Architecture," Microelectronics Journal, vol. 41, pp. 639-650, 2010.

[10] Constantinos Efstathiou, N. Moshopolous, N. Axelos, K. Pekmestzi, Efficient Modulo 2n+1 Multiply and MultiplyAdd Units Based on Modified Booth Encoding, Integration, the VLSI Journal, 47 (2014), pp. 140-147.

[11] Manas Ranjan Meher, Ching Chuen Jong, and Chip-Hong Chang, "A High Bit Rate Serial-Serial Multiplier with Onthe-Fly Accumulation by Asynchronous Counters”, IEEE trans. On VLSI systems, Vol. 19, No. 10, pp. 1733-1745, October, 2011. 


\section{BIOGRAPHIES OF AUTHORS}

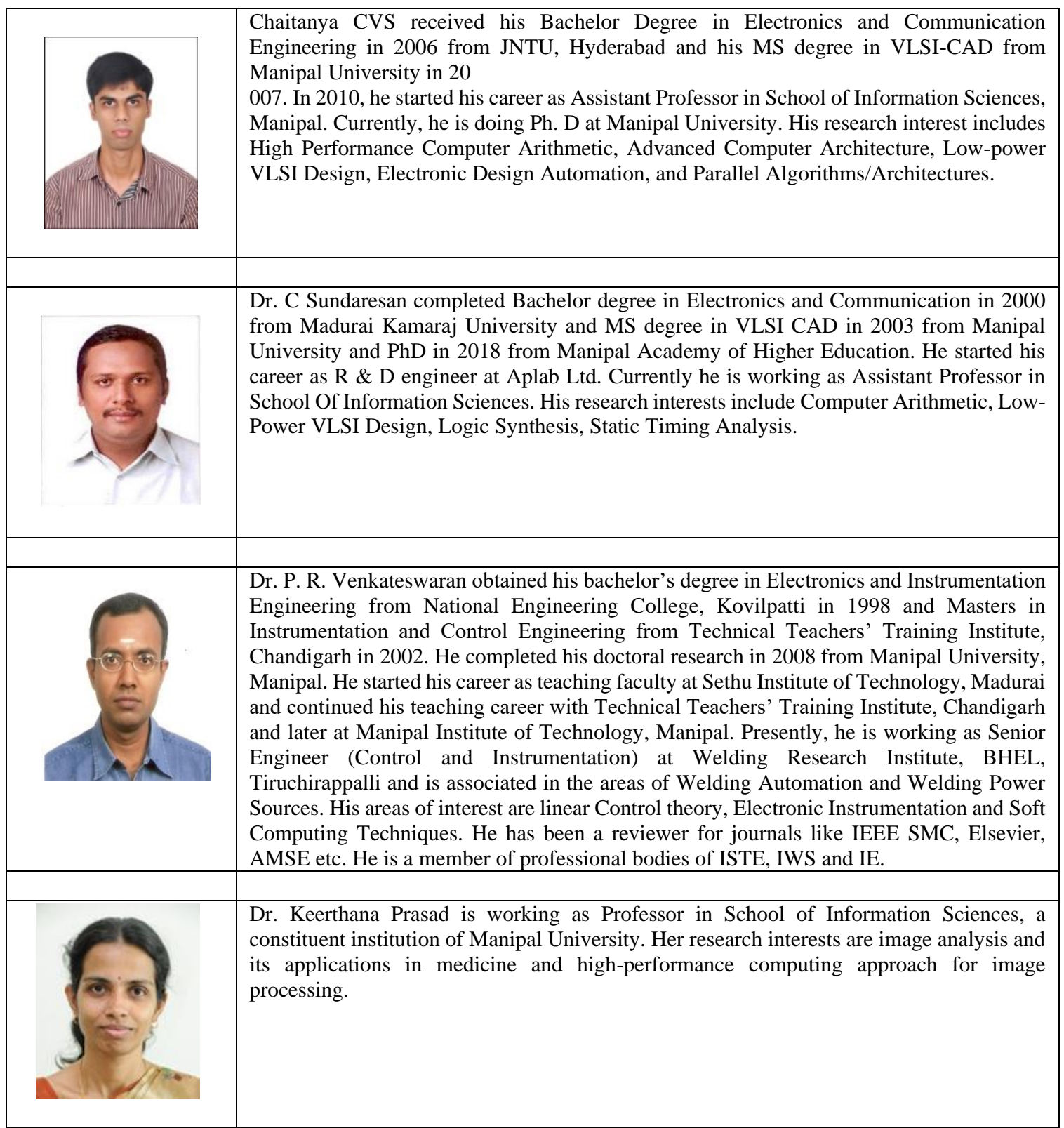

